\title{
Structure of the Wigner $9 j$ Coefficients in the Bargmann Approach
}

Alfred C. T. Wu

Deparlment of Physics, Universily of Michigan, Ann Arbor, Michigan 48104

(Received 19 June 1971)

Bargmann's treatment of the Clebsch-Gordan $(3 j)$ and Racah $(6 j)$ coefficients is here extended to the case of Wigner $9 j$ coefficients. The generating function for the $9 j$ coefficient is computed by the analytic method. The result is compared to the Schwinger's expression derived with the algebraic (boson operator) method. The full symmetry of the Wigner $9 j$ coefficients is manifest and transparent in the Bargmann's formalism. A new explicit expression for the Wigner $9 j$ coefficient is derived as a sixfold sum which may be regarded as the analog of the Racah's formula for the Racah coefficient.

\section{INTRODUCTION}

The practical implication in the use of the rotation group machinery in handling the composition of angular momentum states obviously warrants a detailed analysis of the coupling and recoupling coefficients such as the Clebsch-Gordan, Racah, and Wigner $9 j$ coefficients. Bargmann's beautiful exposition of the representations of the rotation group ${ }^{1}$ in the Wigner Festschrift issue of the Reviews of Modern Physics (October, 1962) is presumably well known. It is quite remarkable that results on the representations and the coupling and recoupling coefficients all come out in such a coherent, transparent and effortless way. We have in mind here, in particular, the following features of the coupling and recoupling coefficients: (i) derivation of the generating functions; (ii) symmetry properties; and (iii) explicit summation expressions. For the Clebsch-Gordan $(3 j)$ and Racah $(6 j)$ coefficients, these features are all worked out in detail in the Bargmann article. For the Wigner $9 j$ coefficients, ${ }^{2}$ the standard reference for the generating function is the celebrated unpublished report by Schwinger, ${ }^{3}$ where everything is obtained by the algebraic (boson operator) method. The 72-element symmetry for the $9 j$ symbol is discussed by Jahn and Hope. ${ }^{4}$ For an easy access to many classic references as well as a highly readable introductory account of the quantum theory of angular momentum, the reader is referred to the volume edited by Biedenharn and Van Dam. 5

It would seem desirable, at least for methodological interest, to push the Bargmann scheme to treat the case of the Wigner $9 j$ coefficients. Indeed we find that the analytic approach is sufficiently powerful to render the treatment feasible.

Thus the generating function for the $9 j$ symbol is computed (Sec. II). The result is essentially equivalent to that of Schwinger. ${ }^{6}$ In Sec. III, the $9 j$ coefficient is extracted by expansion from the generating function. In Sec.IV, the symmetry of the $9 j$ coefficient is easily read off in the Bargmann formalism. Since the Regge symmetry, ${ }^{7}$ which boosts the previously known symmetry of Clebsch-Gordan and Racah coefficients by a factor of six (from 12 to 72 elements for the former and from 24 to 144 elements for the latter), is entirely contained in the generating function approach, ${ }^{8}$ there does not seem to be an obvious addition to the known 72 element symmetry for the $9 j$ symbols. The fact that the $9 j$ coefficients possess less symmetry than the Racah coefficients may be due to the tighter structure of the former.

In Sec. V, the fifteenfold sum expression for the $9 j$ coefficient in Sec.III is reduced, after proper consideration of the constraint conditions, to a sixfold sum. This new explicit form [Eq. (65) or (65a) below] which manifests full symmetry of the $9 j$ coefficient may be regarded as the analog of the well-known single-sum expression for the Racah coefficient. As one of the consistency checks, it is explicitly verified that when one of the $9 j$ values approaches zero, the sixfold sum for the $9 j$ coefficient collapses to a single sum for the Racah coefficient with the correct phase as well as normalization factors. Finally, the following question is posed. Inasmuch as the Clebsch-Gordan coefficient may be regarded as a ${ }_{3} F_{2}$ function at $x=-1$, and the Racah coefficient as a Saalschutzian ${ }_{4} F_{3}$ function at $x=1$, the seeming inference that the Wigner $9 j$ coefficient might also belong to some ${ }_{p} F_{q}$ function turns out to be unwarranted.

\section{GENERATING FUNC'TION FOR THE WIGNER $9 j$ COEFFICIENT}

As is well known, the Wigner $9 j$ symbol is the recoupling coefficient which connects two different schemes of adding up four angular momenta (such as from $l s$ to ji couplings). ${ }^{9}$ In parallel to Bargmann's treatment of the Racah coefficient where the $6 j$ symbol is defined through a sixfold sum of the product of four $3 j$ symbols, ${ }^{10}$ our starting point here is that the Wigner $9 j$ symbol is defined through a ninefold sum of the product of $\operatorname{six} 3 j$ symbols $^{11}$ :

$$
\begin{array}{r}
\left.\begin{array}{lll}
j_{11} & j_{12} & j_{13} \\
j_{21} & j_{22} & j_{23} \\
j_{31} & j_{32} & j_{33}
\end{array}\right\}=\sum_{\text {all } m_{i j}}\left(\begin{array}{lll}
j_{11} & j_{12} & j_{13} \\
m_{11} & m_{12} & m_{13}
\end{array}\right) \quad\left(\begin{array}{lll}
j_{21} & j_{22} & j_{23} \\
m_{21} & m_{22} & m_{23}
\end{array}\right)\left(\begin{array}{lll}
j_{31} & j_{32} & j_{33} \\
m_{31} & m_{32} & m_{33}
\end{array}\right) \\
\times\left(\begin{array}{ccc}
m_{11} & m_{21} & m_{31} \\
j_{11} & j_{21} & j_{31}
\end{array}\right) \quad\left(\begin{array}{ccc}
m_{12} & m_{22} & m_{32} \\
j_{12} & j_{22} & j_{32}
\end{array}\right)\left(\begin{array}{ccc}
m_{13} & m_{23} & m_{33} \\
j_{13} & j_{23} & j_{33}
\end{array}\right) .
\end{array}
$$

It is clear that there are six triangular relations, one for each triplets of $j$ belonging to the rows and columns on the left-hand side of Eq. (1). The following short-hand notations are found convenient:

$$
J_{p} \equiv \sum_{q=1}^{3} j_{p q}, \quad p=1,2,3
$$




$$
\begin{aligned}
K_{p} & \equiv \sum_{q=1}^{3} j_{q p}, \\
k_{p q} & \equiv J_{p}-2 j_{p q}, \quad p, q=1,2,3, \\
k_{p q}^{\prime} & \equiv K_{q}-2 j_{p q} .
\end{aligned}
$$

There are a number of constraints on the $18 k$ and $k^{\prime}$, namely

$$
\begin{aligned}
& \sum_{q} k_{p q}=J_{p}, \\
& \sum_{p} k_{p q}^{\prime}=K_{q}, \\
& k_{p q}-k_{p q}^{\prime}=J_{p}-K_{q}, \\
& \sum_{p} J_{p}=\sum_{p} K_{p} \equiv J .
\end{aligned}
$$

The generating function $\rho\left(\tau, \tau^{\prime}\right)$ for the $9 j$ symbol will be written as

$$
\rho\left(\tau, \tau^{\prime}\right)=N \sum_{k_{p q}} \sum_{k_{p q}^{\prime}}\{9 j\} \prod_{p . q} \tau_{p q}^{k_{p q}} \tau_{p q}^{\prime_{p q}^{\prime \prime}},
$$

where the normalization constant

$$
N \equiv\left[\frac{\prod_{p}\left(J_{p}+1\right) !\left(K_{p}+1\right) !}{\prod_{p, q} k_{p q} ! k_{p q}^{\prime} !}\right]^{1 / 2}
$$

comes from the six sets of triangular coefficients. On account of Eq. (1), the generating function can be readily cast into the integral representation

$$
\begin{aligned}
\rho\left(\tau, \tau^{\prime}\right) & =\int d \mu_{18}\left(\zeta_{p q}\right) \prod_{p=1}^{3} \Phi_{p} \prod_{q=1}^{3} \tilde{\Phi}_{q} \\
& =\int d \mu_{18}\left(\zeta_{p q}\right) \exp \sum_{p=1}^{3}\left(D_{p}+\tilde{D}_{p}\right),
\end{aligned}
$$

where the six $\Phi$ 's are the generating functions for the $3 j$ symbols, one for each triplet in Eq. (1). Explicitly, we have $\mathrm{e}^{12}(p, q=1,2,3)$

$\Phi_{p} \equiv \Phi\left(\tau_{p 1}, \tau_{p 2}, \tau_{p 3} ; \zeta_{p 1}, \zeta_{p 2}, \zeta_{p 3}\right)=\exp D_{p}$,

$\tilde{\Phi}_{q} \equiv \Phi\left(\tau_{1_{q}}^{\prime}, \tau_{2 q}^{\prime}, \tau_{3 q}^{\prime} ; \Gamma^{T} \bar{\zeta}_{1 q}, \Gamma^{T} \bar{\zeta}_{2 q}, \Gamma^{T} \bar{\zeta}_{3 q}\right)=\exp \tilde{D}_{q}$,

where $\zeta_{p q}$ is a shorthand notation for the two-component complex

$$
\zeta_{p q} \equiv\left(\begin{array}{c}
\xi_{p q} \\
\eta_{p q}
\end{array}\right)
$$

Recall that the $\xi$ and $\eta$ are the variables that enter in the basis function

$$
v_{m}^{j}(\zeta)=\frac{\xi^{j+m} \eta^{j-m}}{\sqrt{(j+m) !(j-m) !}} .
$$

In Eq. (10b), $\Gamma^{T}$ is the transpose of $\Gamma \equiv\left(\begin{array}{ll}0 & -1 \\ 1 & 0\end{array}\right)$, and $\bar{\zeta}$ is the complex conjugate of $\zeta$. The $D$ are $3 \times 3$ determinants, namely

$$
D_{p}=\left|\begin{array}{ccc}
\tau_{p 1} & \tau_{p 2} & \tau_{p 3} \\
\xi_{p 1} & \xi_{p 2} & \xi_{p 3} \\
\eta_{p 1} & \eta_{p 2} & \eta_{p 3}
\end{array}\right|
$$

$$
\tilde{D}_{q}=\left|\begin{array}{ccc}
\tau_{1 q}^{\prime} & \tau_{2 q}^{\prime} & \tau_{3 q}^{\prime} \\
\bar{\eta}_{1 q} & \bar{\eta}_{2 q} & \bar{\eta}_{3 q} \\
-\bar{\xi}_{1 q} & -\bar{\xi}_{2 q} & -\bar{\xi}_{3 q}
\end{array}\right|
$$

Finally by $d \mu_{N}(z)$ is meant the "Gaussian" measure in the space of $N$-dimensional complex variables, namely

$$
d \mu_{N}(z)=\pi^{-N} \exp (-\bar{z} \cdot z) d^{N} z .
$$

The proof for Eq. (9) is obvious and will be omitted here. 13

The computation of the generating function for the $9 j$ symbol is thus reduced to the evaluation of the righthand side of Eq. (9). This can be done in three steps.

Step 1: Integrate over the variables $\zeta_{1 q}$. Only four of the six $D^{\prime}$ s, namely $D_{1}, \widetilde{D}_{q}, q=1,2,3$, contribute here. Write

$$
\sum_{q} \tilde{D}_{q}=\sum_{q}\left(c_{q} \bar{\xi}_{1 q}+d_{q} \bar{\eta}_{1 q}\right)+E
$$

where

$$
\begin{aligned}
c_{q} & \equiv \tau_{3 q}^{\prime} \bar{\eta}_{2 q}-\tau_{2 q}^{\prime} \bar{\eta}_{3 q}, \\
d_{q} & \equiv-\tau_{3 q}^{\prime} \bar{\xi}_{2 q}+\tau_{2 q}^{\prime} \bar{\xi}_{3 q}, \\
E & \equiv \sum_{q} \tau_{1 q}^{\prime} \bar{\delta}_{2 q, 3 q},
\end{aligned}
$$

with

$$
\delta_{p q, p^{\prime} q^{\prime}} \equiv\left|\begin{array}{ll}
\xi_{p q} & \xi_{p^{\prime} q^{\prime}} \\
\eta_{p q} & \eta_{p^{\prime} q^{\prime}}
\end{array}\right|
$$

$$
\begin{gathered}
\int d \mu_{6}\left(\zeta_{11}, \zeta_{12}, \zeta_{13}\right) \exp \left(c \cdot \bar{\xi}_{1}+d \cdot \bar{\eta}_{1}\right) \\
\times \exp \left(D_{1}+E\right)=\exp f, \\
f=\left|\begin{array}{lll}
\tau_{11} & \tau_{12} & \tau_{13} \\
c_{1} & c_{2} & c_{3} \\
d_{1} & d_{2} & d_{3}
\end{array}\right|+E .
\end{gathered}
$$

Step 2: Integrate over the variables $\zeta_{2 q}$. Rewrite

$$
f=\left|\begin{array}{lll}
\sigma_{1} & \sigma_{2} & \sigma_{3} \\
\bar{\xi}_{21} & \bar{\xi}_{22} & \bar{\xi}_{23} \\
\bar{\eta}_{21} & \bar{\eta}_{22} & \bar{\eta}_{23}
\end{array}\right|+\left(c^{\prime} \cdot \bar{\xi}_{2}+d^{\prime} \cdot \bar{\eta}_{2}\right)+E^{\prime},
$$

where

$\sigma \equiv\left(\begin{array}{ccc}\tau_{11} & \tau_{32}^{\prime} & \tau_{33}^{\prime} \\ \tau_{12} & \tau_{31}^{\prime} & \tau_{33}^{\prime} \\ \tau_{13} & \tau_{31}^{\prime} & \tau_{32}^{\prime}\end{array}\right)$

$c_{p}^{\prime} \equiv \sum_{q} H_{p q} \bar{\eta}_{3 q}$

$d_{p}^{\prime} \equiv-\sum_{q} H_{p q} \bar{\xi}_{3 q}$

$H \equiv\left(\begin{array}{ccc}\tau_{11}^{\prime} & -\tau_{13} \tau_{22}^{\prime} \tau_{31}^{\prime} & \tau_{12} \tau_{23}^{\prime} \tau_{31}^{\prime} \\ \tau_{13} \tau_{21}^{\prime} \tau_{32}^{\prime} & \tau_{12}^{\prime} & -\tau_{11} \tau_{23}^{\prime} \tau_{32}^{\prime} \\ -\tau_{12} \tau_{21}^{\prime} \tau_{33}^{\prime} & \tau_{11} \tau_{22}^{\prime} \tau_{33}^{\prime} & \tau_{13}^{\prime}\end{array}\right)$,

$E^{\prime} \equiv\left(\lambda \times \bar{\xi}_{3}\right) \cdot \bar{\eta}_{3}$, 
with

$$
. \lambda \equiv\left(\begin{array}{ccc}
\tau_{11} & \tau_{22}^{\prime} & \tau_{23}^{\prime} \\
\tau_{12} & \tau_{21}^{\prime} & \tau_{23}^{\prime} \\
\tau_{13} & \tau_{21}^{\prime} & \tau_{22}^{\prime}
\end{array}\right)
$$

Since $E^{\prime}$ and $D_{3}$ are independent of the variables $\zeta_{2 q}$, the relevant expression is

$$
\begin{aligned}
\exp \left(E^{\prime}\right. & \left.+D_{3}\right) \int \prod_{q} d \mu_{3}\left(\xi_{2 q}\right) \exp \left(c^{\prime} \cdot \bar{\xi}_{2}\right) \\
& \times \int \prod_{q} d \mu_{3}\left(\eta_{2 q}\right) \exp \left(d^{\prime} \cdot \bar{\eta}_{2}\right) \exp \left[\left(\sigma \times \bar{\xi}_{2}\right) \cdot \bar{\eta}_{2}\right] \exp D_{2} \\
= & \exp \left(E^{\prime}+D_{3}\right) \int \prod_{q} d \mu_{3}\left(\xi_{2 q}\right) \exp \left(c^{\prime} \cdot \bar{\xi}_{2}\right) \exp X,
\end{aligned}
$$

where

$$
X \equiv d^{\prime \prime} \cdot \xi_{2}+\bar{\xi}_{2}^{T} A \xi_{2}
$$

with

$$
d_{q}^{\prime \prime} \equiv\left(d^{\prime} \times \tau_{2}\right)_{q}
$$

$A=\left(\begin{array}{ccc}\sigma_{2} \tau_{22}+\sigma_{3} \tau_{23} & -\sigma_{2} \tau_{21} & -\sigma_{3} \tau_{21} \\ -\sigma_{1} \tau_{22} & \sigma_{1} \tau_{21}+\sigma_{3} \tau_{23} & -\sigma_{3} \tau_{22} \\ -\sigma_{1} \tau_{23} & -\sigma_{2} \tau_{23} & \sigma_{1} \tau_{21}+\sigma_{2} \tau_{22}\end{array}\right)$

The last integral in Eq. (28) can be performed with the aid of Bargmann's theorems on the Laplacian integrals. ${ }^{15}$ The result is

$$
\begin{aligned}
\int \Pi d \xi_{2 q} \exp \left[-\bar{\xi}_{2}^{T}(1-A) \xi_{2}+c^{\prime} \cdot \bar{\xi}_{2}+d^{\prime \prime} \cdot \xi_{2}\right] \\
=\operatorname{det}(1-A)^{-1} \exp d^{\prime \prime} T(1-A)^{-1} c^{\prime}
\end{aligned}
$$

with

$$
\operatorname{det}(1-A)=\left(1-\sigma_{1} \tau_{21}-\sigma_{2} \tau_{22}-\sigma_{3} \tau_{23}\right)^{2} .
$$

Last Step: Integrate over the remaining variables $\zeta_{3 q}$. The remaining expression is

$$
\begin{aligned}
\rho \equiv \operatorname{det}(1-A)^{-1} \int d \mu_{3}\left(\xi_{3 q}\right) d \mu_{3}\left(\eta_{3 q}\right) \\
\times \exp \left[E^{\prime}+D_{3}+d^{\prime \prime} T(1-A)^{-1} c^{\prime}\right] .
\end{aligned}
$$

The last term in Eq. (34) can be rewritten as

$$
d^{\prime \prime} T(1-A)^{-1} c^{\prime}=\bar{\xi} \frac{T}{3} P \eta_{3}
$$

in which $c^{\prime}$ is given by Eq. (23), $d^{\prime \prime}$ by Eq. (30) and

$$
\begin{aligned}
&(1-A)^{-1}= {[\operatorname{det}(1-A)]^{-1 / 2} } \\
& \times\left(\begin{array}{ccc}
1-\sigma_{1} \tau_{21} & -\sigma_{2} \tau_{21} & -\sigma_{3} \tau_{21} \\
-\sigma_{1} \tau_{22} & 1-\sigma_{2} \tau_{22} & -\sigma_{3} \tau_{22} \\
-\sigma_{1} \tau_{23} & -\sigma_{2} \tau_{23} & 1-\sigma_{3} \tau_{23}
\end{array}\right), \\
& P \equiv[\operatorname{det}(1-A)]^{-1 / 2} H^{T}\left(\begin{array}{ccc}
0 & \tau_{23}-\tau_{22} \\
-\tau_{23} & 0 & -\tau_{21} \\
\tau_{22} & -\tau_{21} & 0
\end{array}\right) H .
\end{aligned}
$$

Equation (34) then becomes (dropping now the subscripts 3 on the $\zeta$ 's)

$$
\begin{aligned}
\rho= & \operatorname{det}(1-A)^{-1} \int d \mu_{3}(\xi) d \mu_{3}(\eta) \\
& \times \exp \left[(\lambda \times \bar{\xi}) \cdot \bar{\eta}+\left(\tau_{3} \times \xi\right) \cdot \eta+\bar{\xi}^{T} P \bar{\eta}\right] \\
= & \operatorname{det}(1-A)^{-1} \int d \mu_{3}(\xi) \exp \left[\left(\lambda \times \bar{\xi}+\bar{\xi}^{T} P\right) \cdot\left(\tau_{3} \times \xi\right)\right]
\end{aligned}
$$

$$
\begin{aligned}
& =\operatorname{det}(1-A)^{-1} \int d \mu_{3}(\xi) \exp \bar{\xi} T_{\Omega} \xi \\
& =\operatorname{det}(1-A)^{-1} \cdot \operatorname{det}(1-\Omega)^{-1}
\end{aligned}
$$

where

$$
\Omega_{p q}=\left(\lambda \cdot \tau_{3}\right) \delta_{p q}-\tau_{3 p} \lambda_{q}+Q_{p q}
$$

with

$$
Q \equiv P\left(\begin{array}{ccc}
0 & -\tau_{33} & \tau_{32} \\
\tau_{33} & 0 & -\tau_{31} \\
-\tau_{32} & \tau_{31} & 0
\end{array}\right)
$$

The final result is remarkably simple. The generating function for the $9 j$ symbol reads

$$
\begin{aligned}
\rho\left(\tau, \tau^{\prime}\right) & =N \sum_{k, k^{\prime}}\{9 j\} \prod_{p, q} \tau_{p q}^{k}{ }^{k} \tau_{p q}^{\prime k_{p q}^{\prime}} \\
& =\operatorname{det}(1-A)^{-1} \cdot \operatorname{det}(1-\Omega)^{-1} \\
& \equiv\left[G\left(\tau, \tau^{\prime}\right)\right]^{-2}
\end{aligned}
$$

in which $\operatorname{det}(1-A)^{-1}$ is given by (33) and $\operatorname{det}(1-\Omega)=\left[1-\tau_{31}\left(\lambda_{1}+P_{23}\right)-\tau_{32}\left(\lambda_{2}+P_{31}\right)\right.$

$$
\left.-\tau_{33}\left(\lambda_{3}+P_{12}\right)\right]^{2} \text {. }
$$

With the substitution of the matrix elements $P_{i j}$ from (37), we get

$$
G\left(\tau, \tau^{\prime}\right)=1-\sum_{p, q=1}^{3} a_{p q}-\sum_{\alpha=1}^{6} b_{\alpha},
$$

where

$$
\begin{aligned}
& \left(a_{p q}\right) \\
& \equiv\left(\begin{array}{ccc}
\tau_{21} \tau_{31} \tau_{12}^{\prime} \tau_{13}^{\prime} & \tau_{22} \tau_{32} \tau_{11}^{\prime} \tau_{13}^{\prime} & \tau_{23} \tau_{33} \tau_{11}^{\prime} \tau_{12}^{\prime} \\
\tau_{11} \tau_{31} \tau_{22}^{\prime} \tau_{23}^{\prime} & \tau_{12} \tau_{32} \tau_{21}^{\prime} \tau_{23}^{\prime} & \tau_{13} \tau_{33} \tau_{21}^{\prime} \tau_{22}^{\prime} \\
\tau_{11} \tau_{21} \tau_{32}^{\prime} \tau_{33}^{\prime} & \tau_{12} \tau_{22} \tau_{31}^{\prime} \tau_{33}^{\prime} & \tau_{13} \tau_{23} \tau_{31}^{\prime} \tau_{32}^{\prime}
\end{array}\right) \\
& \left(b_{\alpha}\right) \equiv\left(\begin{array}{c}
\tau_{11} \tau_{11}^{\prime} \tau_{32} \tau_{32}^{\prime} \tau_{23} \tau_{23}^{\prime} \\
\tau_{31} \tau_{31}^{\prime} \tau_{22} \tau_{22}^{\prime} \tau_{13} \tau_{13}^{\prime} \\
\tau_{21} \tau_{21}^{\prime} \tau_{12} \tau_{12}^{\prime} \tau_{33} \tau_{33}^{\prime} \\
-\tau_{11} \tau_{11}^{\prime} \tau_{22} \tau_{22}^{\prime} \tau_{33} \tau_{33}^{\prime} \\
-\tau_{21} \tau_{21}^{\prime} \tau_{32} \tau_{32}^{\prime} \tau_{13} \tau_{13}^{\prime} \\
-\tau_{31} \tau_{31}^{\prime} \tau_{12} \tau_{12}^{\prime} \tau_{23} \tau_{23}^{\prime}
\end{array}\right)
\end{aligned}
$$

Note that the sum of the six $b$ terms may be written as $\operatorname{det}\left|\tau_{p q} \tau_{p q}^{\prime}\right|$, namely,

$$
\sum_{\alpha=1}^{6} b_{\alpha}=-\left|\begin{array}{lll}
\tau_{11} \tau_{11}^{\prime} & \tau_{12} \tau_{12}^{\prime} & \tau_{13} \tau_{13}^{\prime} \\
\tau_{21} \tau_{21}^{\prime} & \tau_{22} \tau_{22}^{\prime} & \tau_{23} \tau_{23}^{\prime} \\
\tau_{31} \tau_{31}^{\prime} & \tau_{32} \tau_{32}^{\prime} & \tau_{33} \tau_{33}^{\prime}
\end{array}\right|
$$

where the indices of the entries in (46) may be regarded as complementary to those in (44).

Comparison with Schwinger's result shows that the Schwinger's expression [Eq. (4.37) of Ref. 3] differs from our Eq. (43) in a few changes of signs and some shifts in the primes. Since our expression [Eqs. (44) and (45)] cannot tolerate such changes without ruining the symmetry of the problem, we believe that these discrepancies are most likely typographical in origin. 


\section{EXPANSION FORMULA FOR THE 9j COEF-} FICIFNT

Expanding $\left[G\left(\tau, \tau^{\prime}\right)\right]^{-2}$ in powers of $\tau$ and $\tau^{\prime}$, we have

$$
\begin{aligned}
N \sum_{k, k \prime}\{9 j\} \prod_{p, q} \tau_{p q}^{k}{ }_{p q} \tau_{\substack{\prime \\
p q q}}^{k} \\
\quad=\sum_{n=0}(n+1)\left(\sum_{p, q=1} a_{p q}+\sum_{\alpha=1}^{6} b_{\alpha}\right)^{n} \\
\quad=\sum_{n=0}(n+1) ! \prod_{p, q=1}^{3} \prod_{\alpha=1}^{6} \frac{a_{p q q}^{\nu}}{\nu_{p q} !} \frac{b_{\alpha}^{\omega_{\alpha}}}{\omega_{\alpha} !} .
\end{aligned}
$$

Comparing the coefficients of $\tau_{p q}$ and $\tau_{p q}^{\prime}$ on both sides of this equation, we get

$$
\left\{9_{j}\right\}=N^{-1} \sum_{\nu, \omega} \frac{(n+1) !}{\prod_{p, q=1}^{3} \prod_{\alpha=1}^{6} \nu_{p q} ! \omega_{\alpha} !}(-1)^{\omega_{4}+\omega_{5}+\omega_{6}},
$$

where the summations on the right-hand side are subject to the following set of matrix component-wise constraints:

$$
\begin{aligned}
k_{p q} & =\hat{\nu}_{p q}+\omega_{p q}, \\
k_{p q}^{\prime} & =\left(\hat{\nu}_{q p}\right)^{T}+\omega_{p q}, \\
n & =\sum_{p, q=1}^{3} \nu_{p q}+\sum_{a=1}^{6} \omega_{\alpha},
\end{aligned}
$$

where

$$
\left(\omega_{p q}\right) \equiv\left(\begin{array}{lll}
\omega_{1}+\omega_{4} & \omega_{3}+\omega_{6} & \omega_{2}+\omega_{5} \\
\omega_{3}+\omega_{5} & \omega_{2}+\omega_{4} & \omega_{1}+\omega_{6} \\
\omega_{2}+\omega_{6} & \omega_{1}+\omega_{5} & \omega_{3}+\omega_{4}
\end{array}\right)
$$

The caret operation on any matrix element $z_{p q}$ is defined here as

$$
\begin{aligned}
\hat{z}_{p q} & \equiv \sum_{p=1}^{3} z_{p q}-z_{p q} \\
& =z_{l q}+z_{m q}, \quad l \neq m \neq p .
\end{aligned}
$$

Note that $k_{p q}, k_{p q}^{\prime}, \nu_{p q}$, and $\omega_{\alpha}$ are all nonnegative integers. With the aid of the identities (4) and (6), we have

$$
\begin{aligned}
& \sum_{q=1}^{3} \nu_{p q}=n-J_{p}, \\
& \sum_{q=1}^{3} \nu_{q p}=n-K_{p} .
\end{aligned}
$$

Thus Eq. (49) reads

$$
\begin{aligned}
& k_{p q}=n-K_{q}-\nu_{p q}+\omega_{p q} \equiv \Re_{p q}, \\
& k_{p q}^{\prime}=n-J_{p}-\nu_{p q}+\omega_{p q} \equiv \Re_{p q}^{\prime} .
\end{aligned}
$$

From Eqs. (3a) and (3b), we get the same expression for $j_{p q}$, namely

$$
2 j_{p q}=-n+J_{p}+K_{q}+\nu_{p q}-\omega_{p q} .
$$

The implication of this statement will be taken up in Sec.V. Before we eliminate those redundant summation variables, let us dispose of the symmetry properties of the $9 j$ coefficient.

\section{SYMMETRY OF THE $9 j$ COEFFICIENT}

The symmetry of the $9 j$ coefficient is embodied in those operations which leave the generating function formally invariant. By formally invariant we include those cases where the determinant [Eq. (46)] may undergo a change of sign, thus resulting in an overall phase factor for the $9 j$ symbol. It will be convenient to speak of the operations on the various sets of nine quantities $j_{p q}, k_{p q}, k_{p q}^{\prime}, \tau_{p q}, \tau_{p q}^{\prime}, \nu_{p q}$, and $a_{p q}$ in terms of their respective $3 \times 3$ matrix arrays. Thus one can easily read off the symmetry from Eqs. (48)(54).

(i) A permutation of given two rows or two columns in the $j$ matrix implies a corresponding permutation in the $k$ and $k^{\prime}$ matrices simultaneously. This induces a corresponding permutation in the $\nu$ and $\omega$ matrices on account of the constraint conditions $\odot k=$ $\odot \varkappa$ and $\odot k^{\prime}=\odot \Re^{\prime}$. The effect on the six $\omega_{\alpha}$ are such that the set $\left(\omega_{1}, \omega_{2}, \omega_{3}\right)$ is mapped onto $\left(\omega_{4}, \omega_{5}\right.$, $\omega_{6}$ ) and vice versa. Thus for an odd number of permutations of rows and columns, there is a net change of phase equal to $(-1)^{\Sigma \omega_{\alpha}}$ which is $(-1)^{J} \equiv(-1)^{\Sigma j_{p q}}$ by virtue of Eqs. (4), (6), (49), and (50).

(ii) Transposition of the $j$ matrix implies $k \leftrightarrow k^{\prime T}$ and hence $\Re \leftrightarrow \Re^{\prime}$, or $\nu \leftrightarrow \nu^{T}$ and $\omega \leftrightarrow \omega^{T}$, (i.e., $\left.\omega_{5} \leftrightarrow \omega_{6}\right)$. This clearly leaves Eq. (48) invariant.

The symmetry (i) and (ii) corresponds to precisely the 72 element symmetry discussed by Jahn and Hope. ${ }^{4}$ As was in the case of $3 j$ and $6 j$ symbols, the Bargmann approach enables one to read off the underlying symmetry in a quite transparent way.

\section{EXPLICIT EXPRESSION FOR THE 9 $j$ COEF- FICIENT}

The expansion formula (48) calls for a sum over 15 variables $\left(\nu_{p q}, \omega_{\alpha}\right)$ [if we disregard $n$ as being fixed by (50)] subject to the constraint conditions (49). Half of the 18 constraints in (49) are actually redundant. The simplest way to see this is to note that the $j$ matrix (54) reconstructed from the $k$ and $k^{\prime}$ matrices turns out to be identical for both (49a) and $(49 \mathrm{~b})$. Thus the number of independent constraints equals the number of the given $j_{p q}$, which is nine in this problem.

We find that, consistent with all the constraint conditions, the 15 summation variables are expressible in terms of a basic set of six independent variables $z_{\alpha}$. The solutions to (49) can be described in general as follows.

We let

$$
\begin{aligned}
& z_{p q} \equiv \nu_{p q}+j_{p q}, \\
& \omega_{p q}^{\prime} \equiv \omega_{p q}+h_{p q},
\end{aligned}
$$

where

$$
\left(h_{p q}\right) \equiv\left(\begin{array}{lll}
h_{1}+h_{4} & h_{3}+h_{6} & h_{2}+h_{5} \\
h_{3}+h_{5} & h_{2}+h_{4} & h_{1}+h_{6} \\
h_{2}+h_{6} & h_{1}+h_{5} & h_{3}+h_{4}
\end{array}\right)
$$

with 16

$$
\begin{array}{ll}
h_{1} \equiv j_{11}+j_{23}+j_{32}, & h_{2} \equiv j_{31}+j_{22}+j_{13}, \\
h_{3} \equiv j_{21}+j_{12}+j_{33}, & h_{4} \equiv j_{11}+j_{22}+j_{33}, \\
h_{5} \equiv j_{21}+j_{32}+j_{13}, & h_{6} \equiv j_{31}+j_{12}+j_{23} .
\end{array}
$$


Then Eqs. (49a) and (49b) are exhausted by

$$
\hat{z}_{p q}+\omega_{p q}^{\prime}=J \text { for all } p, q=1,2,3
$$

by virtue of the identities

$$
\text { and } \begin{aligned}
& J-h_{p q}=k_{p q}+\hat{j}_{p q}=k_{p q}^{\prime}+\left(\hat{j}_{q p}\right)^{T} \\
& \hat{z}_{p q}=\left(\hat{z}_{q p}\right)^{T} \text {. }
\end{aligned}
$$

An explicit solution then calls for a particular choice of (i) $z_{p q}$ in terms of a basic set of $\operatorname{six} z_{\alpha}$ and (ii) $\omega_{\alpha}$ in terms of $\omega_{p q}$. Among many essentially equivalent sets of such solutions, one sufficiently symmetric set is obtained by taking the structure of $z_{p q}$ in exactly the same form as that of the $\omega_{p q}$ of (51), namely

$$
z_{p q}=\left(\begin{array}{lll}
z_{1}+z_{4} & z_{3}+z_{6} & z_{2}+z_{5} \\
z_{3}+z_{5} & z_{2}+z_{4} & z_{1}+z_{6} \\
z_{2}+z_{6} & z_{1}+z_{5} & z_{3}+z_{4}
\end{array}\right)
$$

Then the $\omega_{\alpha}$ may be expressed as

$\omega_{\alpha}=\frac{1}{2}(J-n)+z_{\alpha}-h_{\alpha}, \quad \alpha=1, \ldots, 6$

with

$$
n=\sum_{\alpha=1}^{6} z_{\alpha} \text {. }
$$

The last expression follows from (53) and (55).

We thus get an explicit expression for the $9 j$ coefficient from Eqs. (48), (55), and (63):

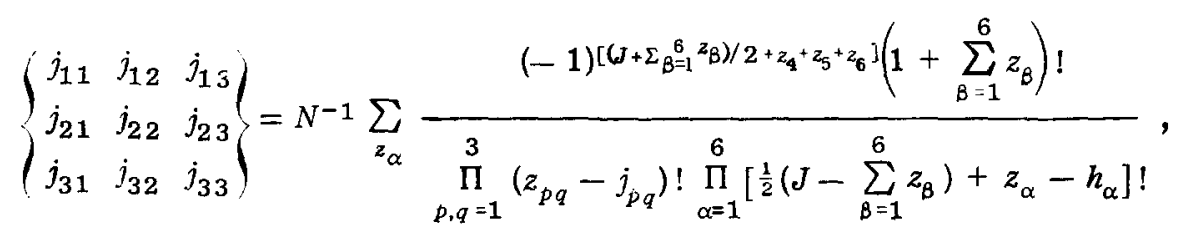

where $z_{p q}$ are given by (62) and the summation is over all $z_{\alpha} \geqslant 0$, such that all the factorial quantities are nonnegative integers.

The symmetry discussed in Sec. IV is of course manifest here. An odd number of permutations of rows and columns of the $j$ matrix which can be compensated by the interchange of the set $\left(z_{1}, z_{2}, z_{3}\right)$ with $\left(z_{4}\right.$, $z_{5}, z_{6}$ ) yields a net change of phase equal to

$$
(-1)^{z_{4}+z_{5}+z_{6}-z_{1}-z_{2}-z_{3}}=(-1)^{\sum \omega_{\alpha}}=(-1)^{J}
$$

by virtue of $(63)$.
We record below two alternate forms which, while manifesting slightly less symmetry than (65), have other redeeming values:

(a) The unpleasant factor of $\frac{1}{2}$ in (65) may be removed by taking in place of (63),

$$
\begin{aligned}
\omega_{p} & =J-n+z_{p}-h_{p}, \\
\omega_{p+3} & =z_{p+3}-h_{p+3},
\end{aligned}
$$

then

$$
\left\{\begin{array}{lll}
j_{11} & j_{12} & j_{13} \\
j_{21} & j_{22} & j_{23} \\
j_{31} & j_{32} & j_{33}
\end{array}\right\}=N^{-1}(-1)^{J} \sum_{z_{\alpha}}^{\sum} \frac{(-1)^{z_{4}+z_{5}+z_{6}}\left(1+\sum_{\beta=1}^{6} z_{\beta}\right) !}{\prod_{p, q=1}^{3}\left(z_{p q}-j_{p q}\right) ! \prod_{p=1}^{3}\left(J-\sum_{\beta} z_{\beta}+z_{p}-h_{p}\right) !\left(z_{p+3}-h_{p+3}\right) !} .
$$

(b) The summation variables $z^{\prime} \mathrm{s}$ in (65) and $\left(65^{\prime}\right)$ may be integers or half-integers (depending on $j_{p q}$ ). By a simple change of basis, the summation variables can be chosen to take on nonnegative integers only. From (65a), let $(p=1,2,3)$

$$
\begin{aligned}
x_{p} & \equiv z_{p}+z_{4}-j_{p p}=\nu_{p p}, \\
x_{p+3} & \equiv z_{p+3}-x_{4}\left(1-\delta_{p 1}\right)-h_{p+3} ;
\end{aligned}
$$

$$
\left\{\begin{array}{lll}
j_{11} & j_{12} & j_{13} \\
j_{21} & j_{22} & j_{23} \\
j_{31} & j_{32} & j_{33}
\end{array}\right\}=N^{-1} \sum_{x_{\alpha} \geq 0} \frac{(-1)^{x_{4}+x_{5}+x_{6}}\left(1+t_{o}+\sum_{\beta=1}^{6} x_{\beta}-x_{4}\right) !}{\prod_{p=1}^{3} x_{p} ! x_{4} !\left(x_{5}+x_{4}\right) !\left(x_{6}+x_{4}\right) ! \prod_{p \neq q=1}^{3}\left(x_{p q}+t_{p q}\right) ! \prod_{p=1}^{3}\left(s_{p}+x_{p}-\sum_{\beta=1}^{6} x_{\beta}\right) !},
$$

where the matrix $\left(x_{p q}\right)$ is formed in terms of $x_{\alpha}$ analogously to the structure of the matrices $\omega_{p q}, z_{p q}$ and $h_{p q}$ before except that $x_{4}$ is now absent along the diagonals. The parameters are given as follows $(p \neq q \neq r=1,2,3)$ :

$$
\begin{aligned}
& t_{p q} \equiv j_{q r}+j_{r p}-j_{p p}-j_{q q}, \\
& t_{0} \equiv J-2 \operatorname{tr} j=\operatorname{tr} k=\operatorname{tr} k^{\prime} \\
& s_{p} \equiv \operatorname{tr} j-j_{q r}-j_{r q} .
\end{aligned}
$$


The following remarks seem in order.

(i) As already pointed out in the beginning of this section, the number of outstanding summation variables equals the number of terms ( $a$ 's and $b^{\prime}$ 's beside the identity term) in the generating function less the number of the $j^{\prime}$ s in the problem. We recall that, for the case of Racah coefficient, such a reduction from a sevenfold to a single sum, as shown by Bargmann, readily yields the well-known Racah's formula. ${ }^{17}$ In this spirit, Eq.(65) may be regarded as the corresponding explicit expression for the $9 j$ coefficient.

(ii) As one of the consistency checks on the expression (65), it is perhaps instructive to verify explicitly the following well known identity ${ }^{18}$ :

$\left\{\begin{array}{lll}j_{11} & j_{12} & j_{13} \\ j_{21} & j_{22} & j_{13} \\ j_{31} & j_{31} & 0\end{array}\right\}=\frac{(-1)^{j_{12}+j_{21}+j_{13}+j_{31}}}{\sqrt{\left(2 j_{13}+1\right)\left(2 j_{31}+1\right)}}\left\{\begin{array}{lll}j_{11} & j_{12} & j_{13} \\ j_{22} & j_{21} & j_{31}\end{array}\right\}$

In establishing (66) directly from (65), we observe that in the limit $j_{33} \rightarrow 0$, the six triangle relations of the $9 j$ symbol collapse into four triangle relations for the $6 j$ symbol, namely $\Phi_{3}$ and $\tilde{\Phi}_{3}$ of Eq. (10) both tend to 1 . This implies that the following limit is to be taken $^{19}:\left(\tau_{31}, \tau_{32}, \tau_{13}^{\prime}, \tau_{23}^{\prime}\right) \rightarrow 0,\left(\tau_{33}, \tau_{33}^{\prime}\right) \rightarrow 1$, and $\tau_{32}^{\prime} \rightarrow \tau_{31}, \tau_{23} \rightarrow \tau_{13}^{\prime}$. The net effect is that in Eqs. (44) and (45), $\left(a_{11}, a_{12}, a_{21}, a_{22}\right) \rightarrow 0$ and $\left(b_{1}, b_{2}, b_{5}\right.$, $\left.b_{6}\right) \rightarrow 0$. This necessitates $\left(\nu_{11}, \nu_{12}, \nu_{21}, \nu_{22}\right) \rightarrow 0$ and $\left(\omega_{1}, \omega_{2}, \omega_{5}, \omega_{6}\right) \rightarrow 0$. The rest of the variables are now expressible in terms of one independent variable, say $x=k_{23}-2 z_{4}$, namely

$$
\begin{aligned}
\left(z_{p q}-j_{p q}\right) & =\left(\nu_{p q}\right) \\
& =\left(\begin{array}{rrr}
0 & 0 & x \\
0 & 0 & J_{1}-J_{2}+x \\
J_{1}-K_{1}+x & J_{1}-K_{2}+x & J_{2}-K_{3}-x
\end{array}\right) \\
n & =J_{1}+x \\
\omega_{3} & =k_{12}^{\prime}-x \\
\omega_{4} & =k_{11}^{\prime}-x
\end{aligned}
$$

In this way, the sixfold sum in (65) is simply reduced to a single sum, and we have

$$
\begin{aligned}
\{9 j\}_{j_{33}=0}= & N^{-1}(-1)^{k_{11}^{\prime}} \sum_{x} \frac{(-1)^{x}\left(1+J_{1}+x\right) !}{x !\left(k_{11}^{\prime}-x\right) !\left(k_{12}^{\prime}-x\right) !\left(k_{23}-x\right) !\left(J_{1}-J_{2}+x\right) !} \frac{1}{\left(J_{1}-K_{1}+x\right) !\left(J_{1}-K_{2}+x\right) !} \\
= & N^{-1}(-1) k_{11}^{\prime} \frac{\left(1+J_{1}\right) !}{k_{11}^{\prime} ! k_{12}^{\prime} ! k_{23} !\left(J_{1}-J_{2}\right) !\left(J_{1}-K_{1}\right) !\left(J_{1}-K_{2}\right) !} \\
& \times_{4} F_{3}\left(2+J_{1},-k_{11}^{\prime},-k_{12}^{\prime}-k_{23} ; 1+J_{1}-J_{2}, 1+J_{1}-K_{1}, 1+J_{1}-K_{2} ; 1\right) .
\end{aligned}
$$

Apart from a normalization constant, the right-hand side is precisely the Racah coefficient, since in our notation we have 17,20

$$
\begin{aligned}
&\left\{\begin{array}{lll}
j_{11} & j_{12} & j_{13} \\
j_{22} & j_{21} & j_{31}
\end{array}\right\} \\
&=\left(\frac{\Pi k_{i j} !}{\prod_{i}\left(J_{i}+1\right) !\left(K_{i}+1\right) !}\right)^{1 / 2} \\
& \times \quad \frac{(-1) J_{1}\left(1+J_{1}\right) !}{\left(J_{1}-J_{2}\right) ! \prod_{i}\left(J_{1}-K_{i}\right) ! k_{11}^{\prime} ! k_{12}^{\prime} ! k_{23} !} \\
& \times{ }_{4} F_{3}\left(2+J_{1},-k_{11}^{\prime},-k_{12}^{\prime},-k_{23} ;\right. \\
&\left.1+J_{1}-J_{2}, 1+J_{1}-K_{1}, 1+J_{1}-k_{2} ; 1\right) .
\end{aligned}
$$

Combining (68) with (69) gives immediately (66) with the correct constant and phase factors as expected.

We note in passing that this ${ }_{4} F_{3}$ function structure for the Racah coefficient in general satisfies a criterion called Saalschutzian 21 [i.e., ${ }_{A} F_{B}\left(a_{i} ; b_{j} ; x\right)$ with $\sum b_{j}=$ $\left.1+\sum a_{i}\right]$. A contrary statement in the literature 22 is traceable to an incorrect transcription of the Racah's formula.

(iii) In the present approach, we have no immediate contact [other than through Eq. (1)] with the other standard formula, namely ${ }^{18}$

$$
\begin{aligned}
& \left\{\begin{array}{lll}
j_{11} & j_{12} & j_{13} \\
j_{21} & j_{22} & j_{23} \\
j_{31} & j_{32} & j_{33}
\end{array}\right\} \\
& =\sum_{j}(-1)^{2 j(2 j+1)}\left\{\begin{array}{l}
j_{11} j_{21} j_{31} \\
j_{32} j_{33} j
\end{array}\right\}\left\{\begin{array}{ll}
j_{12} j_{22} j_{32} \\
j_{21} j & j_{23}
\end{array}\right\}\left\{\begin{array}{ll}
j_{13} j_{23} j_{33} \\
j & j_{11} j_{12}
\end{array}\right\} .
\end{aligned}
$$

We leave it as an open question here whether (65) could yield ( 70 ) directly.

(iv) Finally, it seems desirable to examine the functional structure of the $9 j$ coefficient in the light of known facts on the lower hierarchy. While the Clebsch-Gordan coefficient is a ${ }_{3} F_{2}\left(a_{1}, a_{2}, a_{3} ; b_{1}, b_{2} ; x\right)$ function at $x=-1$, and the Racah coefficient is a ${ }_{4} F_{3}$ $\left(a_{1}, a_{2}, a_{3}, a_{4}: b_{1}, b_{2}, b_{3} ; x\right)$ function at $x=1$, a naive conjecture that the $9 j$ coefficient might also belong to some hypergeometric $F_{q}$ family turns out to be false. The best that can be said in this regard is that the $9 j$ symbol is a folded products of either ${ }_{3} F_{2}$ or ${ }_{4} F_{3}$ functions. Stated otherwise, (65b) can be transcribed into integral representations (of dimensions either six or nine) which can be shown to be quite different from the known representations of a single ${ }_{p} F_{q}$ function.
1 V. Bargmann, Rev. Mod. Phys. 34, 829 (1962). The notation of this paper is followed when possible.
2 E. P. Wigner, famous unpublished 1940 manuscript, reprinted in Ref. 5. 
3 J. Schwinger, famous unpublished 1952 AEC Report, reprinted in Ref. 5.

4 H. A. Jahn and J. Hope, Phys. Rev. 93, 318 (1954).

5 L. C. Biedenharn and H. Van Dam, Eds., Quantum Theory of Angular Momentum (Academic, New York, 1968). A very extensive bibliography on the subject may be found.

6 Apart from some possible misprints in Eq. (4.37) of Ref. 3. See remlarks following Eq. (46) of the present paper.

7 T. Regge, Nuovo Cimento 10, 544 (1958); 11, 116 (1959).

8 See Ref.1.

9 See, e.g., A. R. Edmonds, Angular Momentum in Quantum Mechanics (Princeton U. P., Princeton, N.J., 1957). D. M. Brink and G. R. Satchler, Angular Momentum (Oxford U. P., London, 1968), 2nd ed. Other references may be found in Ref. 5 .

10 Ref. 1, Eq. (4.1)

11 Eq. (1) is quite well known. See, e.g., Ref. 2, Eq. (78b), Ref. 4, Eq. (3), and many standard texts such as Ref. 9 .

12 The particular combination of $\Gamma^{T} \bar{\zeta}$ in Eq. $(10 \mathrm{~b})$ as compared to $\zeta$ in $\mathbf{E q}$. (10a) in essence accounts for the difference in writing the contravariant and covariant indices in the corresponding state vectors. For details, see, e.g., Refs. 1 and 2.
13 The proof is exactly parallel to that leading to Eq. $(4,13)$ of Ref. 1.

14 The integral in Eq. (19) is done exactly the same way as the steps leading to Eq. (4.16) of Ref. 1 .

15 See the Appendix of Ref. 1.

16 It is obvious that the index labeling of $h_{\alpha}$ is in accordance with that of Eq. (45).

17 G. Racah, Phys. Rev. 62, 146 (1942). Cf. Ref. 1, esp.equation following (4.20); and $\mathrm{Eq}$. (69) of the present paper.

18 See any standard textbook, such as Ref. 9

19 Note that $\left(k_{31}, k_{32}, k_{13}^{\prime}, k_{23}^{\prime}\right) \rightarrow 0$ as $j_{33} \rightarrow 0$.

20 It is trivial to transcribe the summation expression of the Racah's formula (Ref. 17) into a hypergeometric function of the ${ }_{4} F_{3}$ type (with argument $x=1$ ).

21 See, e.g. L. J. Slater, Generalized Hypergeometric Functions (Cambridge U. P., Cambridge, 1966), esp. p. 42.

22 D. A. Akyeampong and M. A. Rashid, Trieste Preprint IC/70/134 See Appendix. In their starting point, Eq. (A. 1), $b$ should read $d$ in the next to the last factorial. Hence their last condition (if $b=d$ ) is spurious. Note added in proof: Dr. Akyeampong (private communication) informs the author that this point has been revised in a later version (J. Math. Phys., to appear).

\title{
A Class of Matrix Ensembles*
}

\author{
Freeman J. Dyson \\ Institute for Advanced Study, Princelon, New Jersey 08540 \\ (Received 23 August 1971)
}

\begin{abstract}
A class of random matrix ensembles is defined, with the purpose of providing a realistic statistical description of the Hamiltonian of a complicated quantum-mechanical system (such as a heavy nucleus) for which an approximate model Hamiltonian is known. An ensemble of the class is specified by the model Hamiltonian $H_{0}$, an observed eigenvalue distribution-function $r(E)$, and a parameter $\tau$ which may be considered to be a fictitious "time." Each of $H_{0}, r(E)$, and $\tau$ may be chosen independently. The ensemble consists of matrices $M$ which are obtained from $H_{0}$ by an invariant random Brownian-motion process, lasting for a time $\tau$ and tending to pull the eigenvalues of $M$ toward the distribution $r(E)$. For small $\tau$ the ensemble allows only small perturbations of $H_{0}$. As $\tau \rightarrow \infty$, the ensemble tends to a stationary limit independent of $H_{0}$ and depending on $r(E)$ alone. The following quantitative results are obtained. (1) It is proved that the global eigenvalue distribution in the limit $\tau \rightarrow \propto$ becomes identical with the observed distribution $\gamma(E)$. (2) A nonlinear partial differential equation is obtained for the global eigenvalue distribution $\rho(E, \tau)$ as a function of $E$ and $\tau$. Solution of this equation will show how the distribution changes from the initial form specified by $H_{0}$ at $\tau=0$ to the final form $\nu(E)$ at $\tau=\propto$. Approximate solution shows that deviations of $\rho(E, \tau)$ from $r(E)$ extending over an interval containing $m$ eigenvalues will disappear exponentially as soon as $\tau$ is of the order of $m D^{2}$, where $D$ is the local mean level spacing. (3) Exact analytic expressions are obtained for the correlation functions representing the probabilities for finding $n$ eigenvalues at assigned positions $\left(E_{1}, \ldots, E_{n}\right)$ in the ensemble in the limit $\tau \rightarrow \infty$, irrespective of the positions of the remaining $(N-n)$ eigenvalues. It is made plausible, but not proved, that these correlation functions tend to limits as $N \rightarrow \infty$, which are universal functions independent of $r(E)$. If proved, this statement would imply that the local statistical properties (spacing distributions, etc.) of eigenvalues in the ensemble become, when $\tau$ and $N$ are both large, universal properties independent of the global eigenvalue distributions. In particular, the spacing distributions would be identical with those calculated for more special ensembles by Wigner, Gaudin, and Mehta.
\end{abstract}

\section{THE WIGNER ENSEMBLE}

Wigner ${ }^{1}$ proposed, as a mathematical tool rather than as a physical model for the description of complicated nuclei, the "Gaussian Ensemble" of random matrices. The Gaussian ensemble $E_{G}$ is defined as the set of all real symmetric $(N \times N)$ matrices $M$ with the probability-distribution

$$
p(M)=c \exp \left(-\operatorname{Tr} M^{2} / a^{2}\right)
$$

Here $N$ is any integer, $a$ is a real number, and $c$ is a normalization constant depending on $N$ and $a$. Wigner suggested that in some respects the statistical behavior of the eigenvalues of a matrix $M$ chosen at random in the ensemble $E_{G}$ would mimic the behavior of highly-excited energy levels of a complex nucleus. Since the number of levels of a real nucleus is infinite, the representation of the levels by a finite matrix cannot be complete. It was Wigner's suggestion that the levels of the nucleus and of the random matrix in $E_{G}$ should behave in the same way locally, that is to say, so long as attention is confined to a group of $n$ consecutive levels, where $n$ is a number very small compared to $N$.

One of the first results of the theory of random matrices was Wigner's Semicircle Law. This law ${ }^{2}$ states that the density of eigenvalues per unit energy $E$ of a matrix in the ensemble $E_{G}$ tends to the limit

$$
\begin{aligned}
r(E) & =\left(2 / \pi a^{2}\right)\left(N a^{2}-E^{2}\right)^{1 / 2}, \quad|E|<N^{1 / 2} a, \\
& =0, \quad|E|>N^{1 / 2} a,
\end{aligned}
$$

as $N \rightarrow \infty$. The law was proved by Wigner for a large class of matrix ensembles of which $E_{\mathrm{G}}$ is a special case. The essential requirement upon which the validity of (1.2) depends is that the matrix elements of $M$ be independent random variables.

In spite of its mathematical elegance, the semicircle law is in violent contradiction with the facts of nuclear physics. The level density in real nuclei has roughly the form 